\title{
Krebspatient/-innen bevorzugen orale Antikoagulanzien zur VTE-Therapie
}

Picker N et al. Anticoagulation Treatment in Cancer-Associated Venous Thromboembolism: Assessment of Patient Preferences Using a Discrete Choice Experiment (COSIMO Study). Thromb Haemost 2021; 121: 206-215 doi:10.1055/s-0040-1714739

Eine häufige Komplikation bei Krebserkrankungen ist venöser Thromboembolismus (VTE). Die Therapieentscheidungen für eine Antikoagulation werden vorrangig nach Effektivität und Sicherheit getroffen. Über Präferenzen von Patient/-innen, die auch für die Compliance bedeutsam sind, ist wenig bekannt. Die Studie untersuchte, welche Komfortmerkmale von Antikoagulanzien die Präferenzen von Patient/-innen bestimmen.

Vorherige Richtlinien empfahlen vorrangig niedermolekulares Heparin (NMH) gegenüber Vitamin-K-Antagonisten, die direkten oralen Antikoagulanzien (DOACs) wurden erst kürzlich in die Empfehlungen aufgenommen. DOACs wie Rivaroxaban können einige der Nachteile der anderen Antikoagulanzien abmildern und die gesundheitsbezogene Lebensqualität verbessern. Die COSIMO (Cancer-associated thrOmbo-
SIs - patient-reported outcoMes with rivarOxaban) -Studie untersuchte die Behandlungszufriedenheit von Patient/-innen mit aktiven Krebserkrankungen, die von traditionellen Antikoagulanzien zu Rivaroxaban wechselten. Die komfortbezogenen Präferenzen der Betroffenen für die antikoagulative Therapie wurden als sekundärer Outcome der Studie erhoben. Die COSIMO-Studie war eine multinationale, prospektive, nichtinterventionelle multizentrische einarmige Kohortenstudie. Inklusionskriterium war ein Lebensalter $\geq 18$ Jahre. Picker et al. führten ein DiscreteChoice-Experiment (DCE) innerhalb der COSIMO-Studie durch. Dabei wurden die Patient/-innen gebeten, zwischen hypothetischen Behandlungsoptionen auf der Basis bestimmter Vorgaben zu entscheiden. Die Daten für das DCE wurden mit strukturierten Telefoninterviews zwischen der 4. und 12. Woche nach Aufnahme in die COSIMO-Studie erhoben. Dabei wurde die Kombination folgender Attribute vorgegeben:

- Aufnahmeweg (Injektion oder Tabletten),

- Häufigkeit der Medikation (1- oder 2mal täglich),

- Notwendigkeit regelmäßiger INR-Kontrollen mindestens alle 3-4 Wochen (ja oder nein),

- Interaktionen mit Lebensmitteln oder Alkohol (ja oder nein) sowie

- die Entfernung zur behandelnden Ärztin oder zum behandelnden Arzt (1 versus $20 \mathrm{~km}$ ) als zusätzliches neutrales Merkmal.

Die Auswertung des Datensatzes erfolgte mit der konditionalen Logit-Regression, die alle Attribute als unabhängige Variable enthielt.

In die DCE-Analyse wurden 163 Patient/innen (49,1\% Frauen) im mittleren Alter 
von 63,7 (SD 10,7) Jahren aufgenommen. Sie wurden in Australien, Belgien, Kanada, Dänemark, Frankreich, Deutschland, Italien, den Niederlanden, Spanien und dem Vereinigten Königreich rekrutiert. Die Befragten zogen eine orale Gabe deutlich gegenüber Selbstinjektionen vor. Die Bedeutung dieses Merkmals für die gesamten Behandlungsentscheidungen lag bei $73,8 \%$. Auch eine Medikation ohne diätetische Einschränkungen wurde bevorzugt, die Bedeutung für die Behandlungsentscheidungen betrug 11,8\%. Eine kürzere Entfernung zur behandelnden Ärztin oder zum behandelnden Arzt beeinflusste die
Therapieentscheidungen weniger bedeutsam mit 7,2\%. Die Bedeutung 1-maliger im Vergleich zu 2-maliger Dosis pro Tag betrug 6,5\%. Die Notwendigkeit regelmäBiger INR-Kontrollen mindestens alle 3-4 Wochen erwies sich für die Therapieentscheidungen als kaum bedeutsam $(0,7 \%)$; dabei fanden sich allerdings Unterschiede zwischen den Kontinenten. Während in Kanada oder Australien eher eine Behandlung ohne regelmäßige INR-Kontrollen bevorzugt wurde, war dies in Europa von nur minimaler Bedeutung.

\section{FAZIT}

Ihre Studie zeige, so die Autor/-innen, dass Patient/-innen mit Krebserkrankungen zur Behandlung des venösen Thromboembolismus Antikoagulanzien mit oraler Gabe deutlich bevorzugen. Solche individuellen Präferenzen sollten bei einer Langzeittherapie berücksichtigt werden. So könne die Compliance und damit auch die Effektivität und Sicherheit der Therapie verbessert werden.

Gabriele Dobler, Berlin 